\title{
Prevalencia de las maloclusiones esqueletales en la población de la provincia del
}

\author{
Azuay - Ecuador \\ Prevalence of skeletal malocclusions in the population of the province of Azuay - Ecuador \\ Prevalência de maloclusões esqueléticas na população da província de Azuay - Equador
}

Recibido: 13/04/2021 | Revisado: 19/04/2021 | Acepto: 20/04/2021 | Publicado: 25/04/2021

\author{
Diana Melissa Borja Espinosa \\ ORCID: https://orcid.org/0000-0002-3599-1487 \\ Facultad de Odontología de la Universidad de Cuenca, Ecuador \\ E-mail: diana.borja@ucuenca.edu.ec \\ Emily Antonieta Ortega Montoya \\ ORCID: https://orcid.org/0000-0002-9101-9414 \\ Facultad de Odontología de la Universidad de Cuenca, Ecuador \\ E-mail: emily.ortega@ucuenca.edu.ec \\ Marcelo Enrique Cazar Almache \\ ORCID: https://orcid.org/0000-0002-6806-7442 \\ Facultad de Odontología de la Universidad de Cuenca, Ecuador \\ E-mail: marcelo.cazar@ucuenca.edu.ec
}

\begin{abstract}
Resumen
Objetivo: Establecer la prevalencia de las maloclusiones esqueletales en la población de la provincia del Azuay Ecuador, mediante análisis cefalométricos de radiografías laterales con la finalidad de realizar una comparación con las diferentes regiones del Ecuador. Materiales y métodos: Estudio cuantitativo, descriptivo, retrospectivo y longitudinal en donde se analizó expedientes clínicos de hombres y mujeres de entre 11 y 50 años que acudieron al servicio de cirugía maxilofacial del hospital Monte Sinaí de la ciudad de Cuenca - Ecuador, con diagnóstico de maloclusiones esqueletales. Se obtuvieron 308 expedientes clínicos desde el año 2010 hasta el año 2020, de los cuales 202 fueron incluidos en esta investigación por contar con radiografía cefalométrica del paciente, tomada en el servicio de radiología e imagen del Hospital Monte Sinaí. Resultados: Después del análisis estadístico encontramos que el $49 \%$ de la muestra presenta clase III, $43,56 \%$ clase II y el 7,42\% clase I. Según la base ósea involucrada la clase III puede presentarse como: maxilar normal con prognatismo mandibular (35,64\%), protrusión maxilar con prognatismo mandibular $(22,77 \%)$ o retrusión maxilar con prognatismo mandibular $(13,36 \%)$. Conclusión: La maloclusión esquelética más prevalente en esta población es la clase III y la menos frecuente es la clase I, siendo mayor en hombres que en mujeres en un rango de edad de 16 a 20 años.
\end{abstract}

Palabras clave: Prevalencia; Maloclusiones; Clase esqueletal; Posición maxilar - mandibular.

\begin{abstract}
Objective: To establish the prevalence of skeletal malocclusions in the population of the province of Azuay-Ecuador, through cephalometric analysis of lateral radiographs in order to make a comparison with the different regions of Ecuador. Materials and methods: Quantitative, descriptive, retrospective and longitudinal study in which clinical records of men and women between 11 and 50 years old who attended the maxillofacial surgery service of the Monte Sinaí hospital in the city of Cuenca - Ecuador were analyzed, with a diagnosis of skeletal malocclusions. 308 clinical records were obtained from 2010 to 2020, of which 202 were included in this investigation because they had a cephalometric X-ray of the patient, taken in the radiology and imaging service of the Monte Sinaí Hospital. Results: After statistical analysis, we found that $49 \%$ of the sample was class III, $43.56 \%$ class II and $7.42 \%$ class I. According to the bone base involved, class III can be presented as: maxilla normal with mandibular prognathism (35.64\%), maxillary protrusion with mandibular prognathism (22.77\%) or maxillary retrusion with mandibular prognathism (13.36\%). Conclusion: The most prevalent skeletal malocclusion in this population is class III and the least frequent is class I, being greater in men than in women in an age range of 16 to 20 years.
\end{abstract}

Keywords: Prevalence; Malocclusions; Skeletal class; Maxillary - mandibular position.

\section{Resumo}

Objetivo: Estabelecer a prevalência de maloclusões esqueléticas na população da província de Azuay-Equador, por meio de análises cefalométricas de radiografias laterais a fim de fazer uma comparação com as diferentes regiões do Equador. Materiais e métodos: Estudo quantitativo, descritivo, retrospectivo e longitudinal onde foram analisados prontuários de homens e mulheres entre 11 e 50 anos que compareceram ao serviço de cirurgia maxilofacial do hospital Monte Sinaí na cidade de Cuenca - Equador, com diagnóstico de maloclusão esquelética. Foram obtidos 308 prontuários 
clínicos de 2010 a 2020, dos quais 202 foram incluídos nesta investigação por possuírem radiografia cefalométrica do paciente, tomadas no serviço de radiologia e imagem do Hospital Monte Sinaí. Resultados: Após a análise estatística, constatou-se que 49\% da amostra estava classe III, 43,56\% classe II e 7,42\% classe I. De acordo com a base óssea envolvida, a classe III pode apresentar-se como: maxila normal com prognatismo mandibular $(35,64 \%)$, protusão maxilar com prognatismo mandibular $(22,77 \%)$ ou retrusão maxilar com prognatismo mandibular $(13,36 \%)$. Conclusão: A má oclusão esquelética mais prevalente nessa população é a classe III e a menos frequente é a classe I, sendo maior nos homens do que nas mulheres na faixa etária de 16 a 20 anos.

Palavras-chave: Prevalência; Maloclusões; Classe esquelética; Posição maxilar - mandibular.

\section{Introducción}

La maloclusión es él término empleado para describir desviaciones individuales o combinadas en las relaciones entre los maxilares y los dientes por lo que al mal alineamiento de los dientes se lo conoce como maloclusión dentaria y a la relación inadecuada que puede existir entre el maxilar superior y la mandíbula como maloclusión esquelética (Ash, 1994).

Con el transcurso de los años se ha tenido diferentes clasificaciones. En cuanto a maloclusiones dentales, la primera clasificación fue en 1829 por Samuel Fitch que basó su clasificación en la oclusión de los incisivos superiores con respecto a los inferiores (Vellini, 2002).

En 1842 Carabelli clasificó las maloclusiones dentales en Mordex normalis, Mordex rectus, Mordex apertus, Mordex retrusus y Mordex tortuosus (Vellini, 2002).

En 1899 Angle propuso la clasificación de maloclusiones dentales más utilizada en la actualidad: Clase 1 en donde existe relaciones mesiodistales normales de los maxilares y arcos dentales; Clase 2 división 1: caracterizada por la oclusión distal de los dientes en ambas hemiarcadas de los arcos dentales inferiores; Clase 2 división 2: caracterizada por la oclusión distal de los dientes de ambas hemiarcadas del arco dental inferior, pero con retrusión en vez de protrusión de los incisivos superiores, y Clase 3: caracterizada por la oclusión mesial de ambas hemiarcadas del arco dental inferior (Morales, 2007).

En 1926 Simon clasificó las anomalías según la posición de los arcos dentarios en: Anomalías anteroposteriores: protracción y retracción. Anomalías transversales: contracción y distracción. Anomalías verticales: atracción y abstracción (Quiroz, 1993).

En 1960 Ackerman Y Proffit resuelven los principales puntos débiles del esquema de Angle por lo que basa su clasificación en función de la maloclusión valorando lo siguiente: las proporciones faciales y la estética, la alineación y la asimetría en los arcos dentales, las relaciones esqueléticas dentales en el plano transversal, en el plano anteroposterior y en el plano vertical. (Proffit et al., 2008)

Por otra parte, las maloclusiones esqueletales se presentan por alguna alteración en sus bases óseas. Se puede mencionar que esta puede ser afectada en diferentes aspectos como son en volumen, posición, dirección y forma. Analizando su volumen puede clasificarse en micrognatismo, macrognatismo (anteroposterior, transversal y vertical); según su posición: prognatismo, retrognatismo, dextrognatismo y levognatismo; su dirección: proinclinación, retroinclinación y según su forma: hipergonia e hipogonia (Layana, 2018).

El diagnóstico precoz de las maloclusiones esqueletales y dentales es fundamental a la hora de prevenir, planificar y elaborar tratamientos que permitan interceptar o corregir anomalías dentomaxilares. Una de las herramientas auxiliares más utilizadas para lograr este objetivo es la cefalometría lateral, tanto en la planificación del tratamiento ortodóntico como en la comparación de los resultados y en la valoración del crecimiento del paciente (Sandoval et al., 2011).

La cefalometría como instrumento de diagnóstico en odontología, fue descrita por Broadbent en la primera mitad del siglo XX (Broadbent, 1931), los primeros reportes de su uso clínico los dieron Brodie, Down y Goldstein (Brodie et al., 1938). Desde ese momento las medidas angulares y lineales han sido objeto de estudio por muchos años (De las Rosa et al., 2013) y el desarrollo de los diferentes métodos analíticos y descriptivos darían una nueva dimensión al diagnóstico en la ortodoncia y la 
cirugía maxilofacial ya que la cefalometría ha venido a ocupar un lugar invaluable en la evaluación de las proporciones dentofaciales y clarificar las bases anatómicas de la maloclusión. (Tokunaga et al, 2014). En la década de los 50, se señaló que muchos de los problemas, fracasos y recidivas en tratamientos de ortodoncia, tenían por origen la falta de un análisis cefalométrico completo de las estructuras faciales y óseas (Goldstein, 1953; Koski, 1953; Richardson, 1954; Herzberg, 1954). Entre los análisis cefalométricos, se encuentra el análisis de Downs, el análisis de Steiner, el análisis de Bjork y auxiliares de diagnóstico, como el análisis de Tweed y el análisis de Wits. (Sandoval et al., 2011)

La identificación temprana de los valores cefalométricos de la población adolescente, es sumamente importante y útil, pues la cefalometría radiográfica permite comparar las variaciones en la forma y el tamaño craneofacial de ciertos grupos de población de edades, razas o sexos diferentes. (Bishara, 1971, Kollias \& Krogstad, 1999) En estudios transversales en los que se analiza una determinada población se puede comparar la posición de un determinado hueso o diente o la relación maxilar y extraer datos sobre la tendencia de comportamiento de esas estructuras en esa población. (Menendez, 2013)

La mayoría de los análisis cefalométricos existentes se realizaron en individuos caucásicos, y por lo tanto, la normalidad y prevalencia establecida corresponde a los mismos (Cazar et al., 2019). El trabajo de Chávez confirma las diferencias de valores cefalométricos en la muestra seleccionada y concluye que sí existen diferencias significativas entre los valores de mestizas peruanas con los caucásicos de Ricketts. (Chávez, 2004) Tanto las observaciones encontradas en negros americanos, en indios navajos y en mexicanos varones, se hallaron diferencias significativas, que demuestran que los valores en esta zona de Latinoamérica no se pueden medir con los patrones caucásicos (Menendez, 2013).

En la actualidad contamos con una base de datos con estándares cefalométricos para la población latina. Sin embargo, existe escasa información sobre la prevalencia de las clases esqueletales en el Ecuador y casi nula información en la provincia del Azuay.

Los resultados de esta investigación, proporcionan un parámetro de conocimiento actualizado de la prevalencia de maloclusiones esqueletales en la provincia del Azuay, que puede resultar de utilidad a los odontólogos, con predominio del área de ortodoncia, ortopedia y cirugía maxilofacial, además de establecer diferencias craneométricas con las diferentes regiones del Ecuador.

\section{Metodología}

El presente estudio es cuantitativo, descriptivo, retrospectivo y longitudinal. Para esta investigación se tomaron en cuenta los expedientes clínicos de pacientes hombres y mujeres de entre 11 y 50 años con diagnóstico de maloclusiones esqueletales que acudieron al servicio de cirugía maxilofacial del hospital Monte Sinaí de la ciudad de Cuenca, Ecuador, desde el año 2010 hasta el año 2020. Se revisaron 308 expedientes clínicos de los cuales 106 fueron excluidos por no incluir físicamente la radiografía cefalométrica del paciente o por ser tomada en un centro radiológico distinto al servicio de radiología e imagen del Hospital Monte Sinaí, obteniendo una muestra constituida por 202 expedientes clínicos.

Con la finalidad de evaluar el grado de confiabilidad en la determinación, de las mediciones cefalométricas, se capacitó de forma previa a los investigadores. Las medidas encontradas se compararon con las del experto (interobservador) y con las del investigador (intraobservador) y se determinó la concordancia mediante el coeficiente de Kappa (73,4\% y 76,1\% respectivamente). Los trazos cefalométricos se realizaron con lápices de colores de punta fina marca BIC $®$ y se midieron con regla plástica de $30 \mathrm{~cm}$ marca ARTESCO ${ }^{\circledR}$, con el fin de obtener las siguientes relaciones: cráneo-facial, maxilar-cráneo, mandíbula-cráneo, maxilar-mandíbula, relación dental-maxilar, relación dental-mandíbula y tejidos blandos; sin embargo, para el objetivo de esta investigación se registraron las medidas de: ángulo SNA, SNB, ANB, para establecer la relación maxilarmandíbula- cráneo. 
Los valores cefalométricos fueron digitalizados usando una hoja electrónica en el programa Microsoft Excel versión 2016 ®, y llevadas a fichas específicamente elaboradas para esta investigación, en donde se realizó el análisis de estadística descriptiva, que incluyó análisis de frecuencias, medidas de tendencia central y de dispersión.

\section{Resultados}

En el presente estudio se contó con una muestra de 202 expedientes clínicos de los cuales 93 pertenecen a pacientes de sexo femenino y 109 a pacientes de sexo masculino con un rango de edad de 11 a 50 años y una media de 22.05 años.

Tabla 1. Clases esqueletales según el sexo.

\begin{tabular}{|c|c|c|c|}
\hline \multicolumn{4}{|c|}{ Ángulo ANB } \\
\hline Sexo & Cantidad & Porcentaje & Clase esqueletal \\
\hline \multirow{4}{*}{ Total } & 15 & $7,42 \%$ & Clase I \\
\hline & 88 & $43,56 \%$ & Clase II \\
\hline & 99 & $49 \%$ & Clase III \\
\hline & 202 & $100 \%$ & \\
\hline \multirow{4}{*}{ Mujeres } & 8 & $3,96 \%$ & Clase I \\
\hline & 41 & $20,29 \%$ & Clase II \\
\hline & 44 & $21,78 \%$ & Clase III \\
\hline & 93 & $46,03 \%$ & \\
\hline \multirow{4}{*}{ Hombres } & 7 & $3,46 \%$ & Clase I \\
\hline & 47 & $23,26 \%$ & Clase II \\
\hline & 55 & $27,22 \%$ & Clase III \\
\hline & 109 & $53,96 \%$ & \\
\hline
\end{tabular}

Fuente: Autores.

De acuerdo a la Tabla 1, la maloclusión más frecuente es la clase III representando el 49\%, seguido de la clase II con el 43,56\% y por último la clase I con el 7,42\% del total de la muestra. En el sexo femenino la clase I representa el 3,96\%, la clase II el $20,29 \%$ y la clase III el 21,78\%. En el sexo masculino la clase I representa el 3,46\%, la clase II el $23,26 \%$ y la clase III el $27,22 \%$. 
Tabla 2. Maloclusiones esqueletales según el grupo etario.

\begin{tabular}{|c|c|c|c|c|c|}
\hline \multirow[b]{2}{*}{ Edad } & \multicolumn{3}{|c|}{ ANB } & \multirow[t]{2}{*}{ Total } & \multirow[t]{2}{*}{$\%$} \\
\hline & Clase I & Clase II & Clase III & & \\
\hline \multicolumn{6}{|c|}{ Femenino } \\
\hline $10-15$ & 1 & 6 & 3 & 10 & 4,95 \\
\hline $16-20$ & 2 & 17 & 15 & 34 & 16,83 \\
\hline $21-25$ & 3 & 8 & 9 & 20 & 9,9 \\
\hline $26-30$ & 1 & 3 & 4 & 8 & 3,96 \\
\hline $31-35$ & 1 & 4 & 3 & 8 & 3,96 \\
\hline $36-40$ & 0 & 1 & 6 & 7 & 3,47 \\
\hline $41-45$ & 0 & 1 & 3 & 4 & 1,98 \\
\hline $46-50$ & 0 & 1 & 1 & 2 & 0,99 \\
\hline Total & 8 & 41 & 44 & 93 & 46,04 \\
\hline \multicolumn{6}{|c|}{ Masculino } \\
\hline $10-15$ & 0 & 1 & 2 & 3 & 1,49 \\
\hline $16-20$ & 4 & 22 & 26 & 52 & 25,74 \\
\hline $21-25$ & 2 & 15 & 15 & 32 & 15,84 \\
\hline $26-30$ & 1 & 3 & 7 & 11 & 5,44 \\
\hline 31-35 & 0 & 4 & 2 & 6 & 2,97 \\
\hline $36-40$ & 0 & 1 & 2 & 3 & 1,49 \\
\hline $41-45$ & 0 & 0 & 0 & 0 & 0 \\
\hline $46-50$ & 0 & 1 & 1 & 2 & 0,99 \\
\hline Total & 7 & 47 & 55 & 109 & 53,96 \\
\hline
\end{tabular}

Fuente: Autores.

De acuerdo a la Tabla 2, el grupo etario comprendido entre 16 a 20 años es el que presenta con mayor frecuencia maloclusiones esqueletales clase II (19,30\%) y clase III (20,29\%), seguido del grupo etario comprendido entre 21 a 25 años en donde la clase III es la más prevalente $(11,88 \%)$ con respecto a la Clase II $(11,38 \%)$, esto en el sexo femenino y masculino. 
Tabla 3. Posición de los maxilares según el sexo.

\begin{tabular}{|c|c|c|c|c|c|c|}
\hline \multirow{3}{*}{ Posición de los maxilares } & \multicolumn{4}{|c|}{ Sexo } & \multirow{2}{*}{\multicolumn{2}{|c|}{ TOTAL }} \\
\hline & \multicolumn{2}{|c|}{ Femenino } & \multicolumn{2}{|c|}{ Masculino } & & \\
\hline & $\mathrm{N}$ & $\%$ & $\mathrm{~N}$ & $\%$ & $\mathrm{~N}$ & $\%$ \\
\hline \multicolumn{7}{|c|}{ Maxilar } \\
\hline Protrusión maxilar & 33 & 16,34 & 28 & 13,86 & 61 & 30,2 \\
\hline Retrusión maxilar & 13 & 6,45 & 22 & 10,89 & 35 & 17,34 \\
\hline Maxilar normal & 47 & 23,26 & 59 & 29,2 & 106 & 52,46 \\
\hline TOTAL & 93 & 46,05 & 109 & 53,95 & 202 & 100 \\
\hline \multicolumn{7}{|c|}{ Mandíbula } \\
\hline Prognatismo mandibular & 56 & 27,73 & 89 & 44,06 & 145 & 71,79 \\
\hline Retrognatismo mandibular & 26 & 12,88 & 11 & 5,44 & 37 & 18,32 \\
\hline Mandíbula normal & 11 & 5,44 & 9 & 4,45 & 20 & 9,89 \\
\hline TOTAL & 93 & 46,05 & 109 & 53,95 & 202 & 100 \\
\hline
\end{tabular}

Fuente: Autores.

De acuerdo a la Tabla 3, la posición maxilar más frecuente en el sexo femenino y masculino es la posición normal con $23,26 \%$ y $29,2 \%$ respectivamente, y en el maxilar inferior es el prognatismo mandibular con $27,73 \%$ y $44,06 \%$ respectivamente. La segunda posición más prevalente en el maxilar superior es la protrusión maxilar con 16,34\% en el sexo femenino y 13,86\% en el sexo masculino, y en el maxilar inferior es el retrognatismo mandibular con 16,34\% en el sexo femenino y $12,88 \%$ en el sexo masculino. 
Tabla 4. Base ósea involucrada en la cirugía de acuerdo a la maloclusión.

\begin{tabular}{|c|c|c|c|c|c|}
\hline \multirow{6}{*}{ SNA } & & \multicolumn{3}{|c|}{ SNB } & \multirow[b]{2}{*}{ Total } \\
\hline & & $\begin{array}{l}\text { Prognatismo } \\
\text { mandibular }\end{array}$ & $\begin{array}{c}\text { Retrognatismo } \\
\text { mandibular }\end{array}$ & Mandíbula normal & \\
\hline & Protrusión maxilar & 46 & 5 & 10 & 61 \\
\hline & Retrusión maxilar & 27 & 7 & 1 & 35 \\
\hline & Maxilar normal & 72 & 25 & 9 & 106 \\
\hline & Total & 145 & 37 & 20 & 202 \\
\hline \multicolumn{3}{|c|}{ Posiciones de los maxilares } & $\%$ & \multicolumn{2}{|c|}{ Tratamiento quirúrgico } \\
\hline \multicolumn{3}{|c|}{ Protrusión maxilar-Prognatismo mandibular } & 22,77 & \multicolumn{2}{|c|}{ Retroceso maxilar y mandibular } \\
\hline \multicolumn{3}{|c|}{ Protrusión maxilar-Retrognatismo mandibular } & 2,47 & \multicolumn{2}{|c|}{$\begin{array}{c}\text { Retroceso maxilar con avance } \\
\text { mandibular }\end{array}$} \\
\hline \multicolumn{3}{|c|}{ Protrusión maxilar-Mandíbula normal } & 4,95 & \multicolumn{2}{|c|}{ Retroceso maxilar } \\
\hline \multicolumn{3}{|c|}{ Retrusión maxilar-Prognatismo mandibular } & 13,36 & \multicolumn{2}{|c|}{ Avance maxilar con retroceso mandibular } \\
\hline \multicolumn{3}{|c|}{ Retrusión maxilar-Retrognatismo mandibular } & 3,46 & \multicolumn{2}{|c|}{ Avance maxilar y mandibular } \\
\hline \multicolumn{3}{|c|}{ Retrusión maxilar-Mandíbula normal } & 0,49 & \multicolumn{2}{|c|}{ Avance maxilar } \\
\hline \multicolumn{3}{|c|}{ Maxilar normal-Prognatismo mandibular } & 35,64 & \multicolumn{2}{|c|}{ Retroceso mandibular } \\
\hline \multicolumn{3}{|c|}{ Maxilar normal-Retrognatismo mandibular } & 12,37 & \multicolumn{2}{|c|}{ Avance mandibular } \\
\hline \multicolumn{3}{|c|}{ Maxilar normal-Mandíbula normal } & 4,45 & \multicolumn{2}{|c|}{ No requiere tratamiento quirúrgico } \\
\hline
\end{tabular}

Fuente: Autores.

De acuerdo a la Tabla 4, la posición maxilar combinada menos frecuente es la retrusión maxilar con mandíbula normal $(0,49 \%)$, mientras que las más frecuentes son: maxilar normal con prognatismo mandibular $(35,64 \%)$, protrusión maxilar con prognatismo mandibular $(22,77 \%)$ y retrusión maxilar con prognatismo mandibular (13,36\%), por lo tanto, el tratamiento quirúrgico más realizado es el retroceso mandibular.

\section{Discusión}

El estudio de las maloclusiones esqueletales en pacientes que han culminado su desarrollo es importante en el diagnóstico y planificación del tratamiento interdisciplinario ortodóncico - quirúrgico ya que nos permite conocer la relación sagital de los maxilares basándose en puntos de referencia anatómicos y estructurales (Cisneros et al., 2020), en este estudio se obtuvo como resultado que el patrón esqueletal más frecuente es la clase III esqueletal tanto en el sexo femenino como masculino (49\%), pudiendo presentarse como: maxilar normal con prognatismo mandibular (35,64\%), protrusión maxilar con prognatismo mandibular (22,77\%) o retrusión maxilar con prognatismo mandibular $(13,36 \%)$.

En estudios realizados en distintas provincias del Ecuador, se obtuvo que, en la provincia del Guayas la mal oclusión I esqueletal, tuvo la mayor prevalencia en un 50\%, seguido de la clase II esqueletal en un $43 \%$ y la clase III en un 7\%, además, se determinó que el sexo masculino obtuvo una mayor prevalencia en clase III y I, mientras que el sexo femenino tuvo una mayor prevalencia en la clase II esqueletal. Con respecto a la maloclusión II esqueletal 54\% son a causa de la mandíbula, un $31 \%$ a causa de ambos maxilares y en un 15\% a causa del maxilar superior (Layana, 2018). En la provincia de Pichincha, la maloclusión más frecuente es la Clase II $(78,18 \%)$, seguida de la clase III $(21,87 \%)$ y un valor nulo de clase I (Guerrero, 2014). En la provincia de Chimborazo la maloclusión más frecuente es la Clase II (47,05\%), seguida de la clase I (41,17\%) y por último la clase III (11,76\%) (Sánchez, 2019). 
A nivel de Latinoamérica se encontró que el patrón esqueletal más frecuente en la población mexicana es la Clase I con un 53,3\%, siendo más frecuente en mujeres (64,7\%) en un rango de edad de 13 a 19 años. (De la Rosa et al., 2013). En Perú, el patrón esqueletal predominante en pobladores de 18-25 años es la clase II esqueletal con un 69\%, siendo más frecuente en mujeres (77\%). (Aguilar, 2018). En Brasil la clase III esquelética fue la deformidad más frecuentemente encontrada (81 pacientes), seguida de la clase II (79 pacientes) y la clase I esquelética (11 pacientes). La mayoría de los individuos eran caucásicos (160 pacientes), seguidos de negros (10 pacientes) y solo 1 paciente era asiático. (Boeck et al., 2011)

Diversos son los factores que deben ser evaluados a la hora de determinar un plan de tratamiento entre los que están: el género, la edad, la raza, el biotipo, el pronóstico del desarrollo y la severidad de la discrepancia dentomaxilar. (Sinclair \& Little, 1985)

La motivación de los pacientes para la opción quirúrgica es la esperanza de mejorar su calidad de vida en los siguientes ámbitos: emocional, oral, psicológico y social. La principal influencia fue el aspecto emocional y la menor el funcional. (Eslamipour et al., 2017) Durante el tratamiento combinado ortodóncico - quirúrgico en la etapa prequirúrgica, el malestar psicológico se reduce en los pacientes clase III, mientras que en los pacientes clase II aumenta. Sin embargo, después de la cirugía, los pacientes de ambos grupos muestran mejoras en el malestar psicológico, la discapacidad social y la minusvalía. (Baherimoghaddam, 2016)

\section{Conclusión}

La maloclusión esquelética más prevalente en esta población es la clase III y la menos frecuente es la clase I, siendo mayor en hombres que en mujeres en un rango de edad de 16 a 20 años. Sin embargo, en ambos sexos hubo una combinación de problemas maxilares y mandibulares, que interfirieron directamente en la decisión sobre el plan de tratamiento más adecuado.

Esta investigación se realizó en pacientes que asistieron al servicio de cirugía maxilofacial del Hospital Monte Sinaí de la ciudad de Cuenca-Ecuador, por esta razón los resultados obtenidos se basan en la preocupación de los pacientes por mejorar su estética y estado emocional antes que su condición funcional.

Se requiere realizar nuevos estudios a nivel nacional para obtener medidas cefalométricas estándares de la población ecuatoriana y con ello poder realizar estudios comparativos con otras poblaciones del mundo. La mayoría de estudios realizados sobre los patrones esqueletales basan sus investigaciones en pacientes pediátricos lo que implica que debemos enfocar nuestros esfuerzos en diagnosticar los problemas bucodentales en edades tempranas para dar un tratamiento oportuno y menos agresivo.

\section{Agradecimientos}

Nuestro extensivo agradecimiento a nuestro tutor Dr. Marcelo Cazar por ser una guía durante todo este trabajo y proporcionarnos los expedientes clínicos utilizados para este estudio, además agradecemos el apoyo de la Dra. Jessica Cumbe en el desarrollo de esta investigación; les deseamos éxito en sus labores.

\section{Referencias}

Aguilar Gutiérrez, Y. (2018). Biotipo facial y patrón esqueletal predominante en pobladores de 18-25 años según el análisis cefalométrico de ricketts, en el Distrito de Abancay-2018. (Tesis de grado no publicada). Universidad Tecnológica de los Andes. http://repositorio.utea.edu.pe/handle/utea/138

Ash M.M. (1994). Anatomía dental, fisiología y oclusión de Wheeler. (7ma ed.), Interamericana.

Baherimoghaddam, T., Tabrizi, R., Naseri, N., Pouzesh, A., Oshagh, M., \& Torkan, S. (2016). Assessment of the changes in quality of life of patients with class II and III deformities during and after orthodontic-surgical treatment. International Journal of Oral and Maxillofacial Surgery, 45(4), 476485. https://doi.org/10.1016/j.ijom.2015.10.019

Bishara SE. (1971). Longitudinal cephalometric standards form 5 years of age to adulthood. American Journal of Orthodontic; 79: 35-44. https://doi.org/10.1016/0002-9416(81)90099-3

Boeck, E. M., Lunardi, N., Pinto, A. S., Pizzol, K. \& Boeck, R. J. (2011). Occurrence of skeletal malocclusions in Brazilian patients with dentofacial deformities. Brazilian Dental Journal, 22 (4), 340-345. https://doi.org/10.1590/S0103-64402011000400014 
Broadbent, B. H. (1931). A new X-Ray technique and its application to Orthodontics. The Angle Orthodontist., 1(2):45-66. https://doi.org/10.1043/00033219(1931)001<0045:ANXTAI>2.0.CO;2

Brodie, A.; Downs, W., Goldstein, A \& Myer, E. (1938). Cephalometric appraisal of orthodontic results. The Angle Orthodontist, 8(4):261-5. https://doi.org/10.1043/0003-3219(1938)008<0261:CAOOR >2.0.CO;2

Cazar, M., Piña, V. A. \& Bravo, M. E. (2019). Determinación de estándares Cefalométricos de las diferentes etnias de Ecuador. Revista Latinoamericana de Ortodoncia y Odontopediatría. http://www.ortodoncia.ws/publicaciones/2016/art3.asp

Chávez Matías, E. M. (2004). Valores cefalométricos de una población de escolares peruanos, con oclusión normal, según el análisis lateral de Ricketts. (Tesis de grado no publicada). Universidad Nacional Mayor de San Marcos. http://cybertesis.unmsm.edu.pe/handle/20.500.12672/1724

Cisneros, D., Parise, J. M., Morocho, D., Villarreal, B., \& Cruz, A. (2020). Prevalencia de patrones Máxilo-Mandibulares en pacientes de 8, 5 a 12 años, utilizando Cefalometría de Ricketts en servicios de ortopedia universitarios. Revista KIRU, 17(2). https://doi.org/10.24265/kiru.2020.v17n2.04

De la Rosa Contreras, AV, Bastida, NMM, Ito, TK y Ruiz, IJ (2013). Desarrollo de un estándar cefalométrico para la población mayor de 15 años de la Región Central de México a partir del análisis craneofacial de Ricketts. Revista de la Asociación Dental Mexicana, 70 (5), $251-257$.

Eslamipour, F., Najimi, A., Tadayonfard, A. y Azamian, Z. (2017). Impact of Orthognathic Surgery on Quality of Life in Patients with Dentofacial Deformities, International Journal of Dentistry. https://doi.org/10.1155/2017/4103905

Goldstein, A. (1953). The dominance of the morphological pattern: implications for treatment. The Angle Orthodontist. 23(4):18795. https://doi.org/10.1043/0003-3219(1953)023<0187:TDOTMP>2.0.CO;2

Guerrero Salazar, A. (2014). Determinación del biotipo facial y esqueletal de la población ecuatoriana adulta que visita la Clínica Odontológica de la Universidad San Francisco de Quito con oclusión clase I de Angle utilizando análisis cefalométrico de Ricketts, Steiner y Björk-Jarabak. (Tesis de grado no publicada). Universidad San Francisco de Quito. Obtenido de: https://repositorio.usfq.edu.ec/handle/23000/3866

Herzberg, B. (1954). The Tweed formula anchorage preparation and facial esthetics. The Angle Orthodontist. 24(3):170-7. https://doi.org/10.1043/00033219(1954)024<0170:TTFAPA>2.0.CO;2

Kollias, I., \& Krogstad, O. (1999). Adult craniocervical and pharyngeal changes-a longitudinal cephalometric study between 22 and 42 years of age. Part 1 : morphological craniocervical and hyoid bone changes. The European Journal of Orthodontics, 21(4), 333-344. https://doi.org/10.1093/ejo/21.4.333

Koski, K. (1953). Analysis of profile roentgenograms by means of a newcircle method. Dent. Rec., 73:704-13.

Layana Bernal, A. Y. (2018). Maloclusión esqueletal según Steiner en pacientes de 15-25 años atendidos en la clínica de especialidades INCAFOE en el área de ortodoncia durante el periodo 2016-2018. (Tesis de grado no publicada). Universidad de Guayaquil.

Menéndez Méndez, L. (2013). Estudio comparativo entre mestizas y caucásicos mediante el análisis cefalométrico de Ricketts. Odontol. Sanmarquina; 12(2):6669. http://hdl.handle.net/123456789/3464

Morales, F. J. U. (2007). Clasificación de la maloclusión en los planos anteroposterior, vertical y transversal. Revista de la Asociación Dental Mexicana, 64(3), 97-109.

Proffit W, Fields H, Sarver D. (2008). Ortodoncia Contemporánea. Cuarta edición.

Quiroz O. (1993). Manual de Ortopedia Funcional de los maxilares y Ortodoncia Interceptiva. Actualidades Médico Odontológicas Latinoamérica.

Richardson, J. (1954). Roentgenographic evaluation of orthodontic treatment. The Angle Orthodontist, 24(1):31-7. https://doi.org/10.1043/00033219(1954)024<0031:REOOT>2.0.CO;2

Sánchez Espín, V. C. (2019). Determinación de la clase esqueletal mediante estudios cefalométricos de pacientes con maloclusión. Dental Clinic. Ambato, 2018. (Tesis de grado no publicada). Universidad Nacional de Chimborazo. http://dspace.unach.edu.ec/handle/51000/5412

Sandoval, P., García, N., Sanhueza, A., Romero, A., \& Reveco, R. (2011). Medidas cefalométricas en telerradiografías de perfil de pre-escolares de 5 años de la ciudad de Temuco. International Journal of Morphology, 29(4), 1235-1240. http://dx.doi.org/10.4067/S0717-95022011000400028

Sinclair, P. \& Little, R. (1985). Dentofacial maturation of untreated normals. Am. J. Orthod. Dentofacial Orthop., 88(2):146-56. https://doi.org/10.1016/00029416(85)90239-8

Tokunaga, S., Katagiri, M., \& Elorza, P. H. (2014). Prevalence of malocclusions at the Orthodontics Department of the Graduate School, National School of Dentistry, National University of Mexico (UNAM). Revista odontológica mexicana, 18(3), 175-179.

Vellini F. (2002). Ortodoncia: Diagnóstico y planificación clínica. Editorial Amolca. 